\title{
Musiker bei den Tyrnauer Jesuiten im 18. Jahrhundert
}

\author{
Ladislav KAČIC \\ Bratislava
}

Das Jesuitenkollegium in Tyrnau gehörte im 17. und 18. Jahrhundert zu den bedeutendsten Musiklehranstalten im Königreich Ungarn. Das 1615 zum zweitenmal gegründete Jesuitenkloster mit dem Kollegium (1616) und zwanzig Jahre später auch der Universität (1635) war hinter Wien das zweitbedeutendste Kloster der ganzen Provincia Austriae SJ, wobei der Tyrnauer Rektor den Titel des Vizeprovinzials trug. ${ }^{1}$ Eine der Bedeutung entsprechende, rege Musiktätigkeit der Jesuiten in Tyrnau wurde bis jetzt vor allem von K. Bárdos und R. Rybarič bearbeitet, ${ }^{2}$ beide haben aber ihre Aufmerksamkeit vor allem dem 17. Jahrhundert gewidmet. Die Tätigkeit der Jesuiten auf dem Gebiet des Schuldramas ${ }^{3}$ und des geistlichen Liedes ist teilweise auch schon bekannt. In seiner Arbeit über die Musikinventare der aufgelösten Jesuitenklöster hat K. Kim-Szacsvai auch das Repertoire der Tyrnauer Jesuiten im 18. Jahrhundert in der sog. Kunstmusik gründlich bearbeitet und analysiert. ${ }^{4}$ Unbekannt bleibt aber noch immer, daß bei den Jesuiten in Tyrnau im 18. Jahrhundert viele bedeutende Musiker, ja auch

${ }^{1}$ Vgl. Emil Krapka SJ - Vojtech Mikula SJ (ed.): Dejiny Spoločnosti Ježišovej na Slovensku [Geschichte der Gesellschaft Jesu in der Slowakei]. Cambridge, On.1990, S. 63.

2 Kornél Bárdos: Das Musikleben des Jesuiten- und Piaristen-Ordens in Nordungarn des 17. Jahrhunderts. In: Roczniki teologiczno-kanoniczne, [Theologisch-kanonische Jahrbücher], Tom 34 (1987), S. 315-329; Richard Rybarič: Hudba a hudobný život na Trnavskej univerzite (1635-1777) [Musik und Musikleben an der Tyrnauer Universität]. In: Trnavská univerzita [Tyrnauer Universität], Trnava 1986, S. 46-61; Richard Rybarič: Hudobniny vo fondoch Trnavskej univerzity zo 17. storočia [Musikalien aus dem 17. Jahrhundert in Beständen der Tyrnauer Universität]. In: Hudobný archív [Musikarchiv], 12 (1994), Martin, S. 5-17.

${ }^{3}$ Ladislav Kačic: Die Musik der Jesuitendramen in der Slowakei (1600-1773). Ein Beitrag zur Geschichte der Provincia Austriae SJ. In: Ethnologische, historische und systematische Musikwissenschaft (Oskár Elschek zum 65. Geburtstag), hrsg. von F. Födermayr und L. Burlas. Bratislava 1998, S. 319-333; Ágnes Gupcsó: Musiktheater-Aufführungen an Jesuiten- und Piaristenschulen in Ungarn des 18. Jahrhunderts. In: Studia Musicologica Academiae Scientiarum Hungaricae, Tomus 38 (1997), S. 315-344.

${ }^{4}$ Katalin Kim-Szacsvai: Dokumente über das Musikleben der Jesuiten. Instrumenten- und Musikalienverzeichnisse zur Zeit der Auflösungen. In: Studia Musicologica Academiae Scientiarum Hungaricae, Tomus 39 (1998), S. 283-366. 
mehrere Komponisten studiert haben, u. a. J. Umstatt, A. Renner oder die späteren Franziskaner P. M. Repkovič und P. G. Zrunek sowie J. Vachovský, J. Matejovič u. v. a. Das Ziel des vorliegenden Beitrags ist die persönliche Situation in der Musik am Jesuitenkollegium zu Tyrnau im 18. Jahrhundert zu rekonstruieren, u. zw. aufgrund folgender Quellen: 1. der Studentenmatrikeln aus den Jahren 1695-1773 - Nomenclator gymnasiographus, sive Liber historiarum Gymnasii Societatis Jesu Tyrnaviae (1695-1773), ${ }^{5}$ 2. der gedruckten Periochen und Schuldramentexte (wo sich viele Daten und konkrete Namen befinden), 3. dreier Quellen, die den Personalstatus der Musiker des Jesuitenkollegiums in Tyrnau in den Jahren 1739, 1757 und 1773 widerspiegeln (zwei Musikerverzeichnisse und ein Programm des Faschingskonzertes).

Die ersten Nachrichten über die Musik und Musiker bei den Jesuiten in Tyrnau sind aus der Zeit nach der Gründung des Klosters und des Kollegiums bekannt. Wir fassen nur die für unser Thema wichtigsten Fakten kurz zusammen.

Eine Stiftung für die Ausbildung von etwa 7 Musikern wurde schon 1630 gegründet. ${ }^{6}$ Die Musiker wohnten von 1642 bis $1652 \mathrm{im}$ Seminarium S. Adalberti, dann im Generalseminar. ${ }^{7}$ Noch 1657 wurde für sie ein spezielles Gebäude errichtet, in dem sie bis zur Zeit der großen Umbauten des ganzen Universitätskomplexes (1747-54) wohnten, 1753 wurden sie wieder in einem neuen Gebäude beherbergt. ${ }^{8}$ Aus der „Vorgeschichte“ unseres Themas ist noch wichtig, daß 1642 die gut ausgebildeten Jesuiten-Musiker (,vocalis instrumentalisque musicae insigiter periti“) aus der von Schweden besetzten Olmütz nach Tyrnau geflohen sind. ${ }^{9}$ Sie waren eine Verstärkung des Chors. Es sind viele Fakten über den Ankauf der Noten, Instrumente bekannt, sowie über die konkreten Angelegenheiten, bei welchen die Musik im Tyrnauer Jesuitenkollegium eine wichtige Rolle spielte. ${ }^{10}$ Eine Nachricht über den Ankauf der handschriftlichen Noten, Geigen sowie Trompeten ist noch aus dem Jahr 1747: $:^{11}$ „Pro choro praeter copiosas chartas musicas, bi-

5 Budapest, Egyetemi Könyvtár, Kézirattár [Universitätsbibliothek, Handschriftenabteilung], Ab 122.

6 Bárdos, a.a.O., S. 316. Im Jahr 1678 war das Kapital pro Musica Templi Tyrnaviensis S.J. 4.800 fl, von 1701 bis 1773 7.166 fl. - Rybarič 1994, a.a.O., S. 53.

7 Bárdos, a.a.O., S. 316.

8 Rybarič, a.a.O., S. 53.

9 Bárdos, a.a.O., S. 316.

10 Rybarič 1994, a.a.O., S. 48-52.

11 Ebenda, S. 53 
nae fides, totidem tubae campestres, ac aliae recurveae procuratur" (d. h. Feldtrompeten und Hörner). Zu den größten Gönnern der Musiker gehörte der Rektor des Kollegiums in den Jahren 1672-74, 1687-89 und 1700-01 P. Ladislaus Sennyei. ${ }^{12}$ Jesuiten waren - auch in Tyrnau - im Repertoire immer sehr progressiv orientiert; so wurde schon in den ersten Dezennien des 17. Jahrhunderts in Tyrnau die solistische konzertante Musik der italienischen Komponisten Alessandro Grandi, Ignazio Donati, G. B. Alovisi, Tarquinio Merula, Giovanni Valentini u. a. m. bevorzugt. ${ }^{13}$ Ähnlich war am Ende ihrer unterbrochenen Tätigkeit (1773) ihr Repertoire sehr „,modern“: neben der in der ersten Hälfte des 18. Jahrhunderts wirkenden Johann Georg Zechner, Antonio Caldara, G. J. Donberger u. a. haben Werke des Georg Christoph Wagenseil, Carl Ditters von Dittersdorf oder Joseph und Michael Haydn den „Kern“ des Tyrnauer Musikaliensammlung gebildet. ${ }^{14}$

Aus dem Personalstatus der einzelnen Jesuitenkollegien, darunter auch aus dem Tyrnauer, sind bis jetzt vor allem die Regentes chori (bzw. Praefectes chori) bekannt. ${ }^{15}$ Diese Funktion, die als einzige in der Musik von den Mitgliedern der Gesellschaft Jesu besetzt wurde, war aber direkt mit der Musik, d. h. mit der Musikpraxis, nicht verbunden, es war eher eine organisatorische Funktion wie bei allen Regenten (Seminarii usw.). Regentes chori waren fast ausschließlich Studenten der Theologie, und im Tyrnauer Jesuitenkollegium sind unter ihnen keine bekannten Musiker zu finden. Ein Jesuitenpater oder Laienbruder hatte nämlich viel bedeutendere Pflichten und Aufgaben als jene, die mit der Musik verbunden waren. Ein Mitglied des Jesuitenordens konnte sich der Musik am besten nur in seiner Freizeit widmen, wie z. B. der Laienbruder František Xaver Budinský (1676-1727), der zuerst als Novize, später als Coadjutor, d. h. Ökonome auch im Tyrnauer Kollegium $(1701-09,1726)$ gelebt hat. ${ }^{16}$ Die Musik haben in den Jesuitenkirchen und -kollegien bis auf einige Ausnahmen, wie z. B. Pater J. B. Dolar SJ im 17. Jahrhundert in Wien, von den Jesuiten angestellte weltliche Musiker, vor

12 Ebenda, S. 53-54. P. Ladislaus Sennyei SJ hat z. B. 1675 für den Jesuitenchor die Ausgabe der Messen von Melchior Gletle Expeditionis musicae (Augsburg 1670) besorgt, siehe Jana Kalinayová et al.): Musikinventare und das Repertoire der mehrstimmigen Musik in der Slowakei im 16.-17. Jahrhundert, Bratislava 1995, S. 36.

13 Vgl. den Katalog der Musikalien aus der Bibliothek des Jesuitenkollegiums zu Trnava aus dem Jahr 1632. In: Jana Kalinayová et al. 1995, S. 33-38.

14 Vgl. Katalin Kim-Szacsvai, a.a.O., S. 339-348

15 Vgl. Ladislaus Lukács S.J.: Catalogi personarum et officiorum Provinciae Austriae S.I., Bd. X, Romae 1994, Bd. XI, Romae 1995, passim.

16 Über F. X. Budinský vgl. näher Ladislav Kačic: Kto bol František Xaver Budinský? Úvahy o živote a diele slovenského skladatel'a. [Wer war F. X. Budinsky? Gedanken zum Leben und Werk des slowakischen Komponisten].In: Slovenská hudba [Slowakische Musik] 29 (1993), S. 376-396. 
allem Studenten, besorgt. Diese Problematik ist aber allgemein bis auf einige Ausnahmen ${ }^{17}$ sehr wenig bearbeitet.

Die Zahl der Musiker des Tyrnauer Jesuitenkollegiums war von 7 bis 15. Es gab aber viele Fälle, wo ein Student des Jesuitenkollegiums in einer anderen Kirche als Musiker gewirkt hatte, z. B. in der Kathedrale oder bei den Paulinern, d. h. nicht alle Schüler waren automatisch ,Musici Collegii Academici“ bzw. „Musici Templi Academici“. Auf der anderen Seite haben auch die Jesuiten in Tyrnau vor allem im 18. Jahrhundert viele weltliche Musiker angestellt und bezahlt. Den kompletten Status dieser „Schicht“ der Tyrnauer Jesuitenmusiker ist fast unmöglich zu rekonstruieren. Aus diesen Gründen ist die Liste der Musiker im Anhang kein komplettes Verzeichnis aller bei den Jesuiten in Tyrnau im 18. Jahrhundert wirkenden Musiker. Trotzdem sind diese Angaben hoffentlich wichtig, man kann daraus u. a. die weiteren Fakten, Daten zu den Lebensschicksalen einzelner Studenten und Musiker gewinnen. Nur einige Studenten, die auch Musiker waren, sind in den Jesuitenorden eingetreten, doch mehrere z. B. in den Franziskanerorden; zwischen den Absolventen sind aber auch spätere Regentes chori, Organisten oder einfache Lehrer auf dem Lande.

Wenn wir nun den Musiker-Status des Tyrnauer Kollegiums im 18. Jahrhundert näher beschreiben wollen, wäre es sinnvoll, nur einige bedeutende Persönlichkeiten hervorzuheben. Von den Musikern und Studenten, die Mitglieder der Gesellschaft Jesu geworden sind, ist als erster der aus Mähren stammende JÁN BRUMOVSKÝ (1688-1726) zu nennen. Er hat 1701-08 in Tyrnau sein komplettes Studium absolviert; als Bakkalaureus der freien Künste und der Philosophie sowie Student der Physik (,A.A.L.L.\& Philosophiae Baccal., Physicus") hat er im Schuldrama Hungaria Tertio Christiana seu Bela Rex (1708) gesungen. Im Jahr 1709 ist er in den Jesuitenorden eingetreten und nach dem Noviziat in Wien bzw. Tyrnau sowie Theologiestudium hat er in den verschiedenen Jesuitenkollegien (Neusohl, Kaschau, Skalitz u. a.) als Lehrer, Katechet und Prediger gewirkt, am Ende seines Lebens sogar als Missionar bei den Pálffyschen Regimenten. ${ }^{18}$ Einer

\footnotetext{
17 Wie z. B. Jiří Sehnal: Die Musik an der Jesuiten-Akademie in Olmütz (Mähren) im frühen 18. Jahrhundert. In: Musik des Ostens, Bd. 14, Kassel-Basel-London-New York 1993, S. 65-83; Darina Múdra: Musikrepertoire, ausübende Musiker und Kopisten der Jesuiten- und Piaristenkirche in Trenčín in den Jahren 1733-1859. In: Musicologica slovaca 7(1978), S. 117-170. Klára Várhidi-Renner: Das Musikleben einiger bedeutenden Kirchen in Pest-Buda im 18. Jahrhundert. In: Studia Musicologica, Tomus 34 (19981), S. 53-122.

18 Ladislaus Lukács SJ: Catalogus generalis seu Nomenclator biographicus personarum Provinciae Austriae Societatis Iesu (1551-1773), Pars I, Romae 1987, S. 134.
} 
der wenigen Musiker, die namentlich Sennyei in seinem Diarium erwähnt, ist der Trompeter TERSTYANSKI (1700-01).

Zwischen 1709 und 1714 hat auch ANTONIUS REPKOVIČ (1694-1758), der spätere Franziskaner Pater Marcus, der Autor der Musikreform in der Marianischen Provinz (um 1730) ${ }^{19}$, sein komplettes Gymnasialstudium in Tyrnau absolviert. Im Unterschied zu Brumovský ist sein ganzes Leben mit der Musik eng verbunden, er ist auch als Komponist bekannt. Aus Repkovičs Studienzeit in Tyrnau wissen wir über sein Auftreten (in der Rhetorikklasse) in der Rolle des „Pictors“ im Drama Homagium Religionis (1713). Es war allerdings wahrscheinlich nur eine Sprechrolle.

In mehreren Tyrnauer Schuldramavorstellungen ist JOSEPHUS REPKOVIČ als Bassist nachweisbar: Carolus I (1712), Fraternae in Fratrem Impietatis ultio (1714), Ludi saeculares (1715). Er hat in Tyrnau aber nur Philosophie und Theologie studiert. Gymnasialklassen hat er bei den Piaristen in Priwitz absolviert. ${ }^{20}$

$\mathrm{Zu}$ den besten Studenten und Musikern des Tyrnauer Jesuitenkollegiums in den ersten Dezennien des 18. Jahrhunderts gehörte bestimmt auch ALEXANDER AIGNER. Er studierte hier 1717-19 Poetik und Rhetorik, 1719 ist er in der letzten Klasse sogar zwischen den preisgekrönten Studenten. Noch 1717 in der Klasse der Poetik war Aigner ein Altist (Perioche zum Schuldrama Rarum charitatis et fortitudinis)! Nach seinem Studium ist er Trompeter (mindensten bis 1737) in der Kapelle des Primas I. Esterházy geworden. Schon 1725 hat er als ,Venerabilis Archi-Capituli Strigoniensis Musicus“ im Schuldrama ,Hymenaeus fraude proditus" mitgewirkt.

In den Jahren 1727-30 hat der aus Wien stammende JOSEPH UMSTATT (1711-62) in Tyrnau studiert. Sein Vater Johann Georg Umstatt war Hofmaler der Kaiserin Anna Amalie und 1724-25 hat für die Esterházys in Cseklís gearbeitet. ${ }^{21}$ Sein Sohn studierte bei den Tyrnauer Jesuiten drei Jahre lang (principista, grammatista, syntaxista) und gehörte ebenfalls zu den besten

\footnotetext{
19 Näheres über P. M. Repkovič vgl. Ladislav Kačic: Missa franciscana der Marianischen Provinz im 17. und 18. Jahrhundert. In: Studia Musicolgica Academiae Scientiarum Hungaricae, 33 (1991), S. 11-16 sowie Ladislav Kačic: P. Marcus Repkovič a národnostný spor františkánov mariánskej provincie v rokoch 1727-1735 [P. Marcus Repkovič und der Nationalitätenstreit der Franziskaner der Marianischen Provinz in den Jahren 17271735]. In: Slavica Slovaca, 32 (1997), S. 154-166.

${ }^{20} \mathrm{Im}$ Jahr 1701 hat Jozef Repkovič als Syntaxista im Schuldrama Patriae Amor et Amator Brutus mitgewirkt. Vgl. István Kilián: A magyarországi piarista iskolai szinjátszás forrásai és irodalma 1799-ig [Quellen und Literatur zum Piaristen-Schuldrama in Ungarn bis 1799]. Budapest 1994, S. 80-81. Er ist hier als ,Josephus Repkovicz Sclavus Synt." angeführt. Ob er ein Verwandter des Anton Repkovič ist, bleibt noch offen.

21 Vgl. Mária Pötzl-Malíková: Bývalé súsošie Panny Márie so sv. Štefanom v areáli kaštiela v Bernolákove a jeho tvorca Anton Leidenfrost. [Das ehemalige Gruppendenkmal der Jungfrau Maria mit dem H1. Stephan im Areal des Schlosses in Bernolákovo und sein Schöpfer Anton Leidenfrost]. Ars 1 (1995), S. 41.
} 
Studenten: 1728 ist er zwischen den preisgekrönten Schülern zu finden. Wo Umstatt später Poetik und Rhetorik studiert hat, ist nicht bekannt. Noch während seiner Tyrnauer Studienzeit war er Musiklehrer (,,im Orgelschlagen") beim Grafen Nicolaus Esterházy. ${ }^{22}$ Es ist interessant, daß Umstatt sich damals als „Organista“ untergeschrieben hat. ${ }^{23}$ Seit 1732 war er Musiker (Musicus) in der Kapelle des Primas I. Esterházy, d. h. ein Kollege von Aigner, in den Akten ist er 1732, 1734 und 1736 zu finden (1734 wurde sogar seine Kompositionstätigkeit erwähnt, er bekam ein Buch ,zum komponieren“, d. h. ein Libretto zu vertonen). 1741 ist Umstatt als „Capellmeister“ und „Magister musicae“ bzw. „Praefectus musicae“ oder „Institutor musicae" in die Dienste des Grafen Dietrichstein in Brünn getreten, wo er bis 1747 blieb. ${ }^{24}$ Seine künstlerische Laufbahn führte ihn immer weiter nach oben, bis er schließlich - nach einer Zwischenstation in Dresden (1749-52, „maestro di cappella“ beim Grafen H. Brühl) - der angesehene und hochgeschätzte bischöfliche Hofkapellmeister und -komponist in Bamberg geworden ist. J. Umstatt gehört zu den bedeutendsten und originellsten mitteleuropäischen Komponisten in der 1. Hälfte des 18. Jahrhunderts, sein Schaffen v. a. für Cembalo ist noch immer nicht entsprechend bewertet. ${ }^{25}$ Sicherlich hat er schon als Student auch Musik zu den Schuldramen komponiert. ${ }^{26}$

Aus den 30er Jahren des 18. Jahrhunderts ist ein Dokument überliefert, in welchem konkrete Funktionen der Musiker im Tyrnauer Jesuitenkollegium verzeichnet sind . Auf die Quelle hat J. Šimončič 1978 aufmerksam gemacht und sie „um 1700“ datiert. ${ }^{27}$ Mit Hilfe der Studentenmatrikel, sowie

22 Kornél Bárdos: A tatai Esterházyak zenéje (1727-1846) [Die Musik der Esterházys in Tata 1727-1846]. Budapest 1978, S. 12 sowie Kornél Bárdos: Neue Forschungen zum Musikleben in der Esterházy-Familie in Tata und Csákvár. In. Haydn Jahrbuch, Bd. 10, Wien-Eisenstadt 1978, S. 30.

23 Magyar Országos Levéltár. Az Esterházy család tatai és csákvári levéltára [Ungarisches Landesarchiv. Archiv der Familie Esterházy aus Tata und Csákvár]. P. 197 Familiarum, Fasc. CXXIII. No. 1, f 322, 324, 330-1, 333 .

24 Jiří Sehnal: Die adeligen Musikkapellen im 17. und 18. Jahrhundert in Mähren. In: Studies in Music History presented to H. C. Robbins Landon on his seventieth birthday (ed. by Otto Biba \& David W. Jones), London 1996, S. 201-202.

25 Allein in zwei großen umfangreichen Codices mit Tastenmusik von P. Pantaleon Roškovský sind 8 hochwärtige Partiten für Cembalo Umstatts sowie einige selbständige Cembalo- bzw. Orgelstücke überliefert. Vgl. Ladislav Kačic: Zborníky pre klavesové nástroje Pantaleona Roškovského [Sammlungen für Tasteninstrumente des Pantaleon Roškovsky]. In: Musicologica Slovaca 12 (1988), S. 179.

${ }^{26}$ Es ist eine Musica theatralis von Umstatt (in einer späteren Abschrift aus dem Jahr 1761, leider unvollständig, überliefert).Vgl. Kačic 1998, a.a.O., S. 326-328. Er könnte auch der Autor der Musik zum Drama von G. Jób SJ Fides in regem et patriam (1727) sein, wobei der Chronist des Tyrnauer Jesuitenkollegiums lediglich folgendes bemerkt: ,,musica ab Amphione quodam nostro composita“ (vgl. Géza Staud: A magyarországi jezsuita iskolai színjátékok forrásai 1561-1773 [Quellen zu den Jesuiten-Schuldramen in Ungarn aus der Zeit 1561-1773]. Bd. I, Budapest 1984, S. 158.

27 Jozef Šimončič: K dejinám hudby v Trnave [Zur Geschichte der Musik in Tyrnau]. In: Musicologica Slovaca 8 (1982), S. 237-239. 
der Perioche zum Drama Theodosius (1737) können wir jedoch das Dokument genau auf das Jahr 1739 datieren. In der Matrikel sind zwar gerade für dieses Jahr keine Studentennamen verzeichnet (,nomina Studiosorum per negligentiam inscribere omiserunt"), aber aufgrund eines Vergleichs mit den Jahren 1738 und 1740 kann man eindeutig „1739“ als Entstehungsjahr feststellen. Die Liste der Musiker im Tyrnauer Jesuitenkollegium für dieses Jahr sah also folgendermaßen aus (siehe Faksimile 1): ${ }^{28}$

\section{In Collegio Soc[ieta]tis JESU Tyrnaviensi Musici et reliqua Familia commoratur seqvens Musici Templi}

$\begin{array}{llll}\text { Joannes König } & \text { Tenorista } & \text { Theol. 2dianni } & \text { Moravus } \\ \text { Antonius Schindler } & \text { organista } & \text { Ph[ysi]cus } & \text { Silesita } \\ \text { Paulus Narosni } & \text { Fidicen } & \text { Logicus } & \text { Moravus } \\ \text { Joannes Zelinka } & \text { Bassista } & \text { Logicus } & \text { Moravus } \\ \text { Josephus Ricsáni } & \text { Tubicen } & \text { Logicus } & \text { Hungarus } \\ \text { Antonius Kunst } & \text { Tubicen } & \text { Rhetor } & \text { Moravus } \\ \text { Georgius Christian } & \text { Fidicen } & \text { Poeta } & \text { Moravus } \\ \text { Carolus Vantke } & \text { Fidicen } & \text { Poeta } & \text { Silesita } \\ \text { Josephus Giesser } & \text { Altista } & \text { Grammat. } & \text { Hungarus } \\ \text { Thomas Schneider } & \text { Discantista } & \text { Princip. } & \text { Hungarus } \\ \text { Josephus Banovics } & \text { Altista } & \text { Parvista } & \text { Hungarus } \\ \text { Joannes Schmidt } & \text { Discantista } & \text { Parvista } & \text { Hungarus } \\ \text { Joannes Kardos } & \text { Calcantista } & & \end{array}$

Einige von ihnen haben schon 1737 in dem oben erwähnten Drama Theodosius mitgewirkt: der aus Neu-Teschen stammende Bassist JOHANN KÖNIG ist in der Perioche zu diesem Schuldrama als „Philosophus absolutus " bezeichnet, der aus Eisenstadt stammende JOSEPH GIESSER war damals noch Diskantist und „Principista“, THOMAS SCHNEIDER „Parvista“ und ebenso Diskantist. Merkwürdig ist dabei, daß im 18. Jahrhundert noch immer viele Musiker aus weit entfernten Gebieten in Tyrnau studiert haben. Der Schlesier KARL WANTKE hat z. B. zuerst bei den Jesuiten in Skalitz 1736-37 Grammatik und Syntax studiert und dann ab 1738 in Tyrnau sein Studium fortgesetzt. Die Kontakte beider Jesuitenkollegien waren gewiß auch in der Musik sehr eng.

Auch in den 40er Jahren des 18. Jahrhunderts haben mehrere gute Musiker in Tyrnau studiert, z. B. der Violinist RAPHAEL MILLER oder JOANNES

28 Trnava, Štátny okresný archív [ Staatliches Landesarchiv] MG, Scholastica 1541-1789, Kart. Nr. 1. 


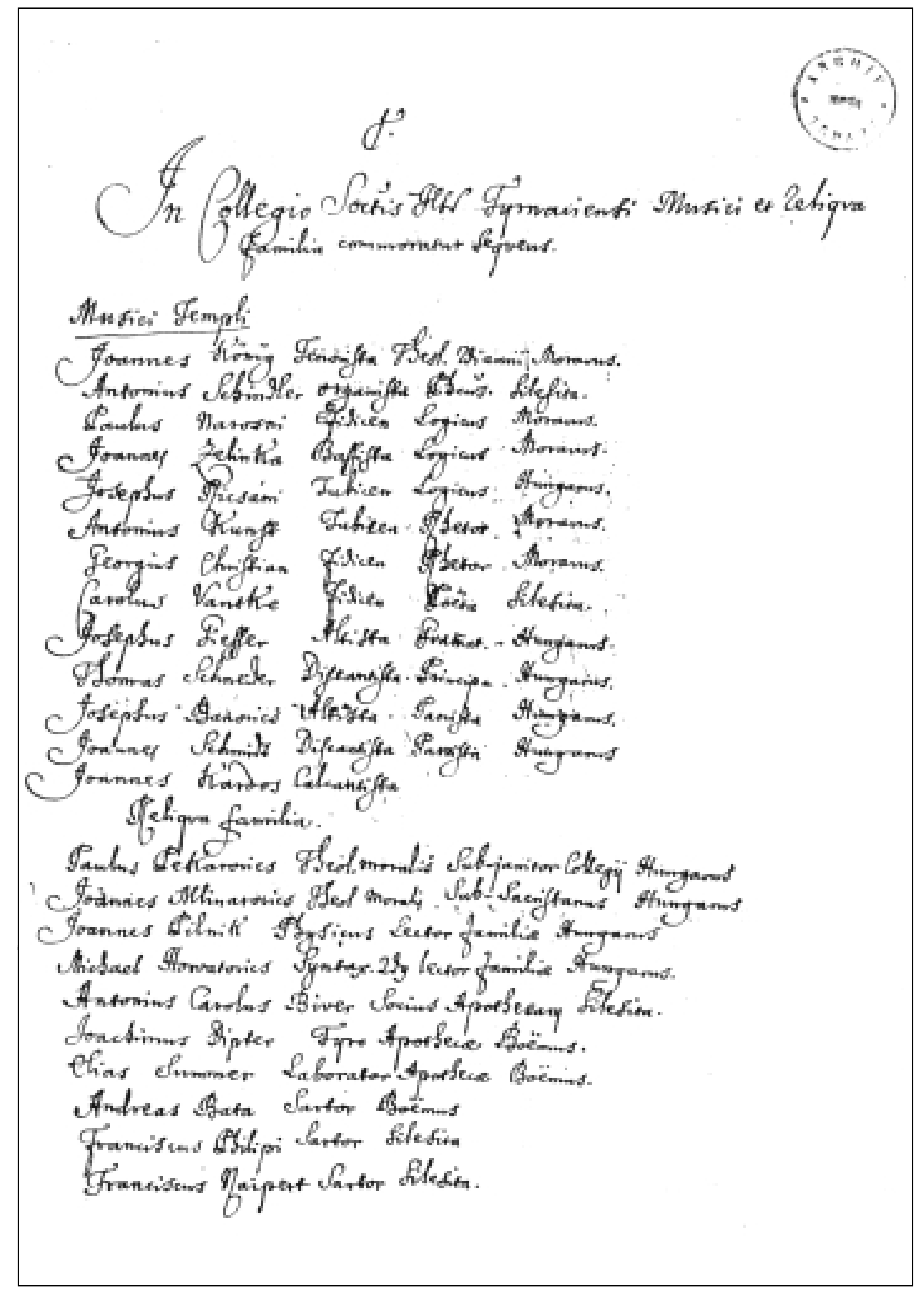

Faksimile 1: Musici Templi [1739] 
REMETHEI (1754-?), der nach Beendigung der Rhetorik bei seinem Eintritt in den Franziskanerorden (1757, Fr. Valvianus Tertiarius) als ,Musicus, organista, Fidicen, Tubicen, cantor Tenorista" bezeichnet wurde. Der beste Musiker der Tyrnauer Jesuiten zu dieser Zeit war aber sicherlich der ausgezeichnete Tenorist und Komponist ANDREAS RENNER (ca. 1729-94), der spätere Schwiegervater von Carl Ditters von Dittersdorf. ${ }^{29}$ Wir wissen nicht, woher er stammte. Er muß schon in seiner Jugend gute musikalische Ausbildung bekommen haben - laut Dittersdorfs Zeugnis war der berühmte Wiener Hofkomponist und Pädagoge Giuseppe Bonno sein Musiklehrer. In den Jahren 1749-50 studierte Renner Poetik und Rhetorik bei den Jesuiten in Tyrnau. Wir wissen nicht, ob er dann auch weiter als Musiker des Kollegiums bzw. der Jesuitenkirche geblieben ist, 1755 finden wir ihn aber als Tenoristen der Preßburger Jesuitenkirche des hl. Erlösers („Tenorista Jesuitarum apud SS. Salvatorem“). Renner dürfte aber bald nach Tyrnau zurückgekehrt sein, denn 1757 war er die „Hauptperson“ eines Faschingskonzertes (siehe weiter unten). 1764 ist Renner Tenorist und Violoncellist der bischöflichen Kapelle in Großwardein geworden und nach deren Auflösung und einem kurzen Aufenthalt in Wien seit 1770 wiederum bischöflicher Sänger, Kammer-Musicus in Johannisberg, Rector scholae und Stadtrat in Jauernig. Andreas Renner war auch ein ausgezeichneter Komponist. Seine gewiß auf den „eigenen Leib“ geschriebenen Werke - Aria de Venerabile Sacramento (,Jesu mel et voluptas”), Aria de pluribus martyribus („Caelestis inter choros $^{6}$ ) sind von höchster Qualität, zugleich aber sehr anspruchsvoll im vokalen Teil, seinen sängerischen Fähigkeiten entsprechend, stilistisch gehören sie zur Frühklassik. Andreas Renner ist bestimmt auch Autor der im Musikalienverzeichnis der Tyrnauer Jesuiten aus dem Jahr 1773 angeführten Werke - einer Symphonie und des Offertoriums de S. Ignatio. ${ }^{30}$ In Tyrnau hat er im Drama Moyses acta ab academicis (1749) als Pluto und Antiope mitgewirkt. In diesem Schuldrama hat auch (die Rolle des Theseus und Maria von Ägypten) ein anderer guter Tenorist - FRANTIŠEK DRINKA (Drienka, ca. 17251763) - gesungen. Nach den Lebensdaten ist nicht klar, ob er mit dem Tenoristen und für kurze Zeit auch Regens chori der Pfarrkirche in Skalitz identisch ist, ${ }^{31}$ Ende der 40er Jahre war er aber Tenorist der Jesuiten in Tyrnau

\footnotetext{
${ }^{29}$ Näheres über ihn siehe Ladislav Kačic: Andreas Renner, Sänger und Komponist, Schwiegervater Dittersdorfs. In: Musikgeschichte Österreichisch-Schlesiens, Untersuchungen und Darstellungen (hrsg. von $\mathrm{H}$. Unverricht, P. Koukal und W. Bein), Würzburg-Opava (im Druck).

30 Vgl. Kim-Szacsvai, a.a.O., S. 364.

31 Vgl. Jozef Šátek: Náboženské pomery v Skalici od reformácie do jozefinizmu [Konfessionelle Umstände in Skalitz von der Reformation bis zum Josephinismus]. Trnava 1946, S. 91-92.
} 
und nach seiner Dienstzeit in der erzbischöflichen Kapelle ${ }^{32}$ seit 1755 bis zum Tode Tenorist der Stadtpfarrkirche zum St. Martin in Preßburg. F. Drinka war außerdem Vater der Sängerin „Nicolina“ (eigentlich Nicolaa Theresia getauft), die nach seinem Tode Andreas Renner adoptierte. ${ }^{33}$ Renner ging mit seiner Stieftochter nach Großwardein und später auch nach Johannisberg, wo sie 1772 die Ehefrau Dittersdorfs geworden ist. F. Drinka könnte übrigens auch kompositorisch tätig gewesen sein. ${ }^{34}$

Aus den 1750er Jahren ist ein wertvolles Dokument über die Musikertätigkeit der Tyrnauer Jesuiten überliefert - ein Programm des am 22. Februar 1757 veranstalteten Faschingskonzerts ${ }^{35}$ (inkl. Texte der Vokalstücke). Zum erstenmal erwähnt dieses Dokument kurz S. Weiss-Nägel SJ, ${ }^{36}$ R. Rybarič hat ihn sogar veröffentlicht, ${ }^{37}$ aber die Analyse fehlt noch immer. Eine „Akademie“" war - entsprechend auch das Schuldrama - die Form einer öffentlichen Präsentation der Schüler eines Jesuitenkollegiums, die sogar in die Ratio studiorum (1586 bzw. 1591) eingegliedert wurde. ${ }^{38}$ Das Dokument hat den folgenden Titel: Academia Musica / Producta a Musicis Collegij Academici S. J. Tyrnaviae / Die 22. Februarij 1757 (siehe Faksimile 2). Faschingskonzerte und -vorstellungen waren in den Jesuitenkollegien sehr beliebt, ${ }^{39}$ und wir besitzen auch mehrere Angaben über solche Vorstellungen in Tyrnau. ${ }^{40}$ Im Falle von solchen Konzerten („Akademien“) war das Programm sehr bunt, aus verschiedenen Stücken - Instrumental- sowie Vokalmusik - zusammengestellt: in diesem Falle waren es Werke von J. G. Zech-

\footnotetext{
32 Ágnes Sas: A pozsonyi Szt. Márton dóm zenés ünnepei és zenészei a 18. században [Musikfeste und Musiker am St. Martinsdom in Preßburg im 18. Jahrhundert]. In: Zenetudományi Dolgozatok 1997-98, Budapest, S. 41, Anm. 89.

33 Näheres siehe Ladislav Kačic: Dittersdorf und die Slowakei (Referate auf dem Symposium zum 200. Todestag Dittersdorfs, Neisse, 23.-27.9.1999), Opole (im Druck).

34 Im Inventar der Jesuitenkirche zu Skalitz (1773) ist auch eine Komposition (Offertorium oder Arie De Beata) mit der Autorenangabe „Brinka“ zu finden. Vg. Kim-Szacsvai, a.a.O., S. 335. Es könnte sich um eine Verballhornung seines Namens handeln.

35 Egyetemi Könyvtár, Kézirattár, Collectio Kaprinayana, XXXIV (Stephani Kaprinai Callectaneorum Tomus XXXIV), S. 640-641.

36 Stanislav Weiss-Nägel SJ: Jezuitské divadlo na Slovensku v XVII. a XVIII. storočí [Das Jesuitentheater in der Slowakei im 17.-18. Jahrhundert]. In: Pamiatke Trnavskej univerzity 1635-1777 [Zum Andenken an die Tyrnauer Universität]. Trnava 1935, S. 295.

37 Richard Rybarič: Dejiny slovenskej hudby I (Stredovek-renesancia-barok) [Geschichte der slowakischen Musik I. Mittelalter-Renaissance-Barock]. Bratislava 1994, S. 71. Dem Programm sind auch Texte der Vokalstücke (Textus ariarum) beigelegt.

38 Vgl. P. E. Krapka SJ - P. V. Mikula SJ, a.a.O., S.229 (als fünfte Stufe der jesuitischen Ausbildungsmethode-1. praelectio, 2. repetitio, 3. disputatio, 4. compositio, 5. academia - ist sie „das öffentliche Auftreten der ausgewählten Studentengruppe, die unter der Führung ihres Professors literarische oder wissenschaftliche Übungen vorträgt“), sowie S. 443 („das öffentliche Auftreten der Schüler eines Kollegiums mit einem festlichen Programm").

39 Vgl. Kačic 1998, a.a.O., S. 321 u. 329-330.

40 R. Rybarič 1998, a.a.O., S. 52 sowie Anm. 29.
} 


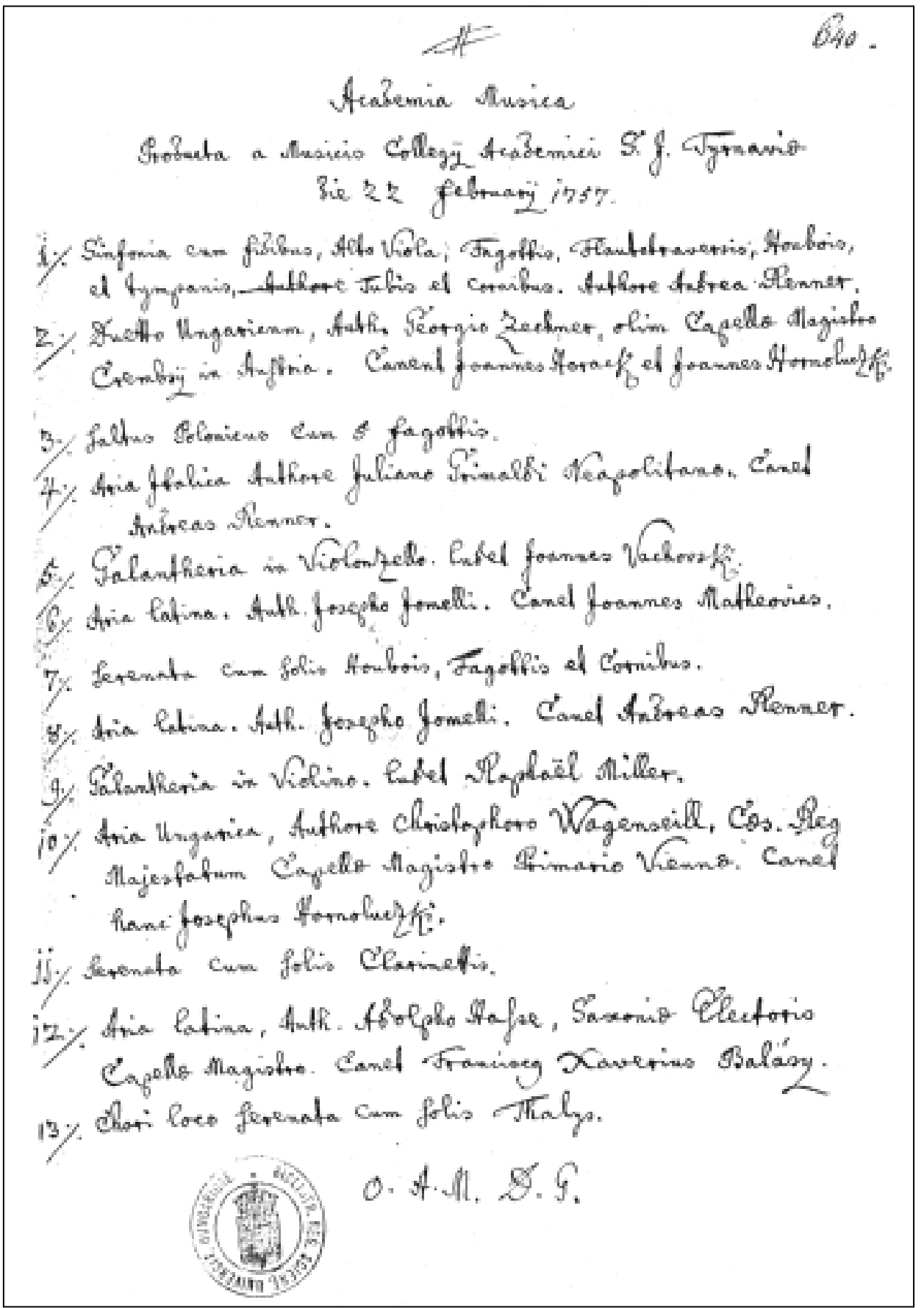

Faksimile 2: Programm des Faschingskonzerts (1757) 
ner, G. Gonelli, G. Grimaldi, G. Ch. Wagenseil, J. A. Hasse und A. Renner sowie anonyme Stücke. Den Anfang bildete eine großbesetzte „Sinfonia cum fidibus, Alto Viola, Fagottis, Flautotraversis, Houbois, et tympanis, Tubis et Cornibus " (d. h. mit allen Instrumenten eines klassizistischen Orchesters) von Andreas Renner - wie schon gesagt, ist sie wahrscheinlich mit jener im Inventar 1773 angegebenen Komposition identisch. Dann folgten wechselweise eine Vokalkomposition mit lateinischem, italienischem und ungarischem Text und Instrumentalkompositionen, manchmal mit einer kuriösen Besetzung, z. B. als Nr. 3 Saltus Polonicus cum 5 Fagottis (!). Neben den solistischen Instrumentalstücken leichteren zeitgenössischen Stils Galantherie für Violine, Violoncello - ist auch Serenata cum Solis Clarinettis zu betonen (es handelt sich höchstwahrscheinlich um die Klarinetten). Den Schluß (,anstatt des Chors") bildete wiederum eine größere, diesmal vokal-instrumentale Komposition - Chori loco Serenata cum Solis Thalys. Es war wahrscheinich eine Komödie mit Musen ${ }^{41}$ (der Autor ist, leider, nicht genannt).

Sehr interessant ist aber die Auswahl und der ,Inhalt" der Vokalkompositionen: das Duetto Ungaricum des Göttweiger Organisten Johann Georg Zechner hat folgenden Text:

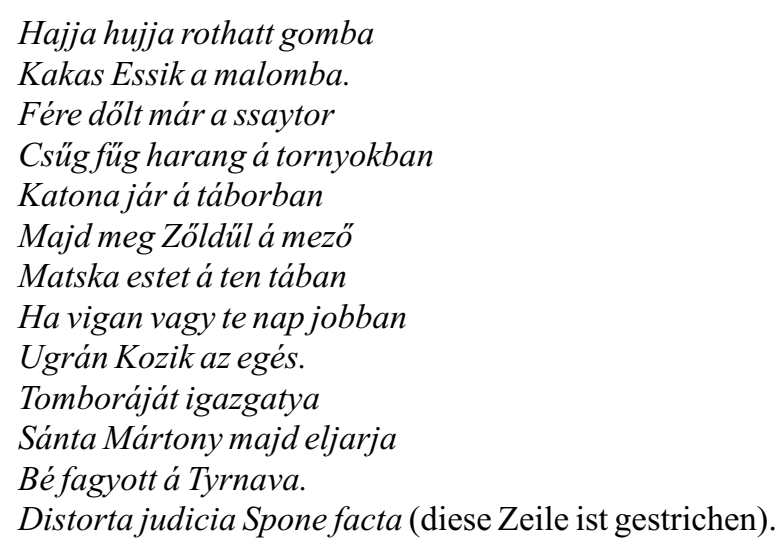

Die erste von Andreas Renner gesungene Aria Italica $2 d a$ (,Se tu potessi o Dio“) des Giuliano Grimaldi hatte einen „seriösen“ Text, die von Ján Matejovič gesungene Aria latina des Giuseppe Gonelli sogar einen liturgischen Text („Textus de omni Sancto“). Die Vokalkompositionen Nr. 10 und

41 „Thalia“ ist „,die Muse der komischen Dichtkunst“, „Thalysia“"war ,ein Fest“”, ,das nach völlig eingesammelten Früchten der Göttin Ceres zu Ehren gefeyert ward“ (Neuestes lateinisch-deutsches und deutschlateinisches Hand-Lexikon, Wien 1842, Bd. 2, S. 630.) 
Nr. 12 haben wiederum einen dem Fasching entsprechenden Text, Nr. 10 mit der Musik von G. Ch. Wagenseil einen makaronischen (lateinischungarischen) Text:

\author{
Bacche addio \\ El múlt áfársang \\ o dolor mio. \\ Vége nyul farkánack \\ Es zsiroß Kolbásznack.
}

Nr. 12 - von Adolf Hasse - hatte wiederum einen lateinischen Text der leichteren Prägung:

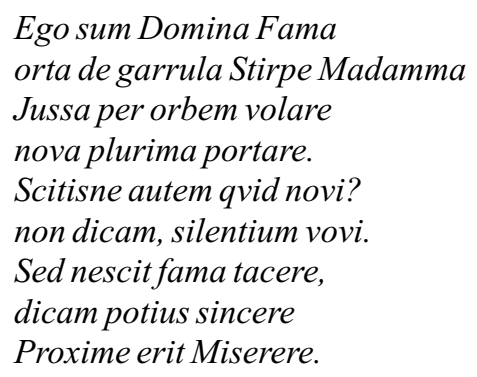

Aufgrund dieses Programmes und der Studentenmatrikel können wir die Besetzung der Musikkappelle der Tyrnauer Jesuiten folgendermaßen rekonstruieren:

$\begin{array}{lll}\text { Andreas Renner } & \text { Tenorista, Fidicen } & \\ \text { Raphael Miller } & \text { Fidicen } & \\ \text { Josephus Vachovsky } & \text { Fidicen } & \text { Rhetor } \\ \text { Joannes Matejovics } & \text { Cantor } & \text { Grammatista } \\ \text { Joannes Hornolucky } & \text { Discantista } & \text { Parvista } \\ \text { Joannes Horak } & \text { Cantor (Altista) } & \text { Grammatista } \\ \text { Franciscus Balássy } & \text { Cantor } & \text { Grammatista }\end{array}$

Außer A. Renner waren ebenfalls zwei andere auch Komponisten: Matejovič und Vachovský. Der aus einer angesehenen Familie stammende JÁN MATEJOVIČ (1741-? $)^{42}$ absolvierte zwischen 1754 und 1761 in Tyrnau sein komplettes Studium (von Parva bis Rhetorik). In der Matrikel ist 1760 bei seinem Namen die Anmerkung „Franciscanus“, aber in den franziskanischen Archivalien ist keine Spur von ihm zu finden. Vielleicht hat er sich in Wien eine gewisse Zeit aufgehalten. Auf dem Titelblatt seiner von Ján Hornolúcky um 1760 abgeschriebenen Sinfonia a 3, eines im Wagenseilischen

42 Näheres über ihn siehe Ján Matejovič: Sinfonia a 3 (hrsg. von L. Kačic), Bratislava 1993. 
Divertimento-Stil komponierten Jugendwerkes, ist nämlich die Anmerkung „Viennae“. JOZEF (JÁN) VACHOVSKÝ ist wiederum Sproß einer weitverzweigten Musikerfamilie. Er hat $1750-57$ bei den Tyrnauer Jesuiten studiert. Ein anderer JOZEF VACHOVSKÝ (1748-?), Sohn des langjährigen Organisten, Ludirector und Notar in Záhorská Bystrica, hat in Trnava nur 1767 (in der Klasse Grammatik) studiert. Im Jahr 1770 ist er in den Franziskanerorden eingetreten (mit dem Namen Fr. Coecilianus), aber noch während des Noviziats ausgetreten (,sponte exivit“). Später war er Organist und Lehrer in Dúbravka (jetzt ein Wohnviertel von Preßburg). ${ }^{43}$ Einige weitere Absolventen des Studiums bei den Tyrnauer Jesuiten sind in den Franziskanerorden eingetreten und weiter dort geblieben, sie wirkten dann lebenslang v. a. als Organisten: Georgius Indrikovič (P. Emericus OFM), Josephus Laurenský (P. Lucianus OFM, bei seinem Namen ist 1765 „Francis.“ angegeben), Stephanus Zetkovič (P. Remigius OFM), Joannes Orema (P. Onuphrius) und Franciscus Hlinický (P. Georgius). Alle waren Mitglieder der Marianischen Provinz. ${ }^{44}$

Von den weiteren Teilnehmern der Faschingsakademie (1757) ist der 1748 aus Buda (nach anderen Angaben 1746 aus Senica) stammende FRANCISCUS XAVERIUS BALÁSSY ${ }^{45}$ nach seinem Studium zwischen 1757 und 1760 in den Jesuitenorden eingetreten (1763). Nach dem Noviziat in Wien (1764-65), dem Studium der Philosophie und Theologie (Leoben, Wien) war er Lehrer und Katechet in Győr (1769), Sopron (1770-72) und Komárno (1773), wo er auch nach der Auflösung des Jesuitenordens als Priester und Lehrer wirkte. F. X. Balássy war auch ein angesehener Schriftsteller ${ }^{46}$ und K. Bárdos gibt an, daß er 1772 in Sopron auch als Regens chori tätig war. ${ }^{47}$

\footnotetext{
43 Welcher Vachovský der Autor der Cembalo-Werke im Musaeum Pantaleonianum des P. P. Roškovský ist, ist schwer zu beurteilen. Es gibt noch weitere Komponisten mit diesem Familiennamen: Ján Bartolomej Vachovský, 1711-1727 Organist in Zohor, der Großvater des letztgenannten, studierte bei den Piaristen in Prievidza und ist Autor eines Veni Sancte Spiritus. Der in der Funktion des Regens chori in Neusohl in der 2. Hälfte des 18. Jahrhunderts wirkende Ján Vachovský komponierte mehrere Kirchenmusikwerke. Vgl. Darina Müdra: Dejiny hudobnej kultúry na Slovensku II (Klasicizmus) [Geschichte der Musikkultur in der Slowakei II]. Bratislava 1993, S. 33; Darina Múdra, Musikalische Klassik in der Slowakei in den Zeitdokumenten, Bratislava 1996, S. 140. Seine Werke sind in Trenčin und Pruské überliefert.

44 Mehrere spätere bedeutende Franziskanermusiker - P. Paulinus Bajan, P. Adalbert Hazakovič u.a. haben am Jesuitenkollegium in Skalitz studiert (Bajan war zuerst Diskantist in der Pfarrkirche, später Tenorist der Jesuitenkirche).

45 Näheres über F. X. Balássy siehe P. Ladislaus Lukács SJ: Catalogus generalis seu Nomeclator biographicus Provinciae Austriae Societatis Iesu (1551-1773), Pars I, Romae 1987, S. 51 sowie Antal Petruch SJ: A trencséni jezsuita noviciátus anyakönyve (1655-1772) [Die Matrikel des Jesuitennoviziats in Trencsén]. Budapest 1942 , S. 182

46 Vgl. Carlos Sommervogel: Bibliothèque de la Compagnie de Jésus, I, Bruxelles-Paris 1891, Sp. 790-91.

47 Kornél Bárdos: Sopron zenéje a 16-18. században [Die Musik in Sopron-Ödenburg im 16.-18. Jahrhundert]. Budapest 1984, S. 378.
} 
In den 50er Jahren studierten auch weitere gute Musiker kurze Zeit in Tyrnau. JURAJ JOZEF ZLATNÍK war eigentlich noch Kollege von A. Renner. 1749-51 hat er Syntax, Poetik und Rhetorik absolviert. Später wurde er Lehrer und Regens chori in Smolenice (ein kleines Städtchen nicht weit von Tyrnau) und ist der Verfasser der Symphonia ex D (1753) für Trompeten, Streichinstrumente und Continuo. ${ }^{48}$ Im Schuljahr 1754 war auch JOZEF ZRUNEK (1736-89) Student der Rhetorik, und noch in demselben Jahr (in der Hälfte des Jahrganges) ist er als „Organista bonus“ in den Franziskanerorden eingetreten und hat den Namen Georgius angenommen. ${ }^{49}$ P. G. Zrunek war der beste Musiker der Salvatorianischen Franziskanerprovinz im 18. Jahrhundert und ist vor allem durch seine zwei originellen Weihnachtsmessen bekannt geworden.

Schließlich steht uns noch ein Dokument zu dem personalen Stand der Musik der Tyrnauer Jesuiten zur Verfügung - aus der Zeit der Auflösung des Ordens 1773. Im Status Personalis et Salarialis Musicorum ${ }^{50}$ sind folgende 15 Musiker aufgezeichnet (die komplette Aufzeichnug siehe Faksimile 3):

Nomina Musicorum

$\begin{array}{ll}\text { Martinus Schaumon } & \text { Primarius } \\ \text { Joannes Fleszer } & \text { Fidicen } \\ \text { Joannes Kolovratek } & \text { Fidicen } \\ \text { Josephus Póts } & \text { Fidicen (Accesista) } \\ \text { Josephus Hoffman } & \text { Fidicen } \\ \text { Marcellus Greska } & \text { Organista } \\ \text { Michael Kunkl } & \text { Huboista } \\ \text { Josephus Millner } & \text { Tubicen } \\ \text { Mathias Kalivoda } & \text { Tubicen } \\ \text { Venceslaus Schorff } & \text { Bassista } \\ \text { Ignatius Kridl } & \text { Tenorista } \\ \text { Franciscus Zelinka } & \text { Discantista } \\ \text { Josephus Langer } & \text { Discantista } \\ \text { Antonius Dvorak } & \text { Altista } \\ \text { Joannes Fabry } & \text { Altista }\end{array}$

\footnotetext{
48 Vgl. Anna Dunajská - Mária Štibrányiová: Hudobný spolok v Trnave 1753-1969, Zbierka hudobnín, Inventár [Der Musikverein zu Tyrnau 1753-1969, Musikaliensammlung, Inventar]. Trnava 1982, S. 24, die Symphonia ex $D$ ist unter dem Inv.-Nr. 1694 zu finden.

${ }^{49}$ Näheres über Zrunek siehe P. Georgius Zrunek OFM: Missa I pro Festis Natalitiis (ex Harmonia pastoralis), hrsg. von L. Kačic, Hudobný fond, Bratislava 1993.

50 Magyar Országos Levéltár [Ungarisches Landesarchiv], E 152 Acta Jesuitica, Irregestrata, Collegium Tyrnaviense 1773, 21/a, S. 175-176.
} 


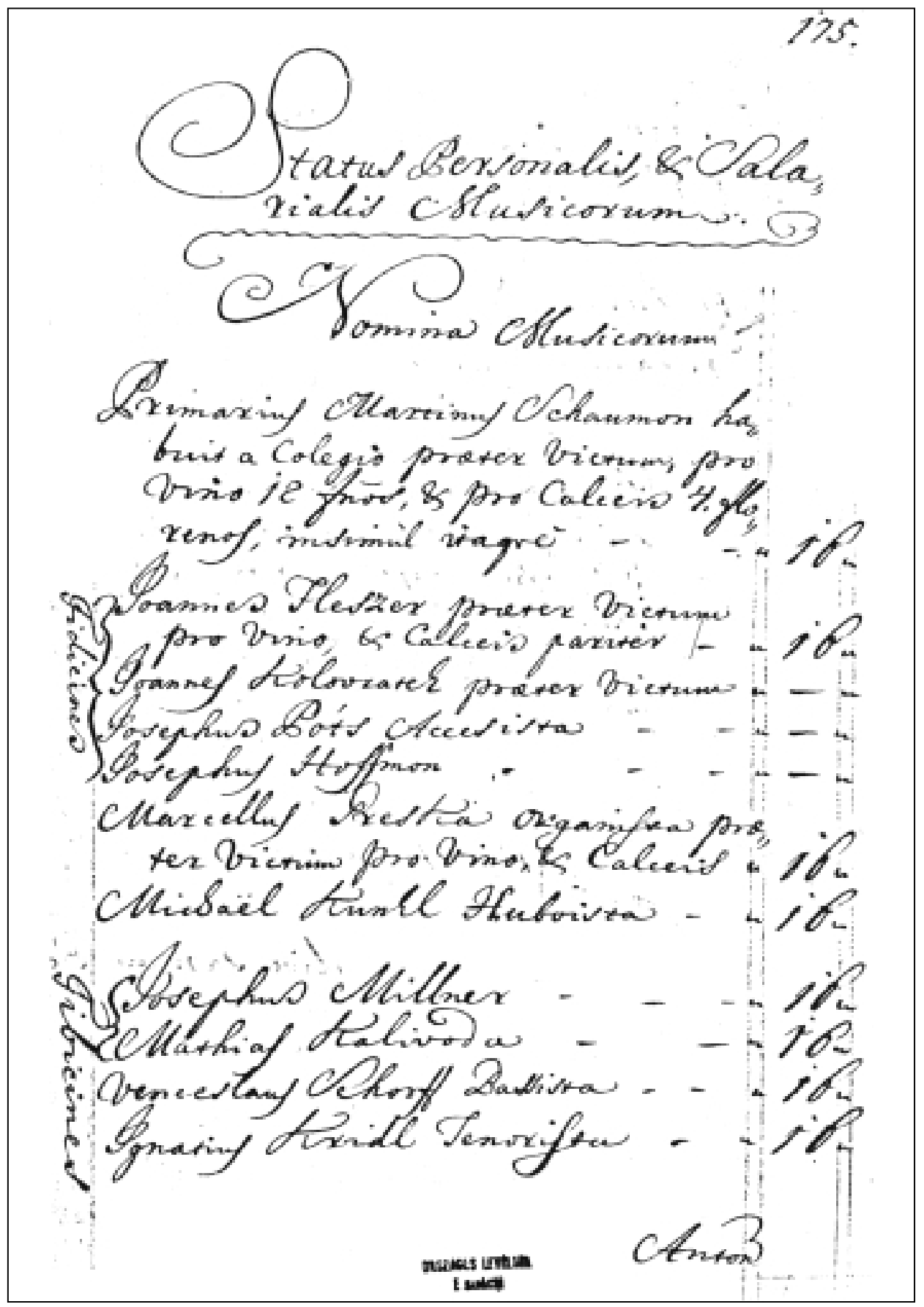

Faksimile 3: Status Personalis et Salarialis Musicorum(1773) 
Von diesen Musikern ist bestimmt der Primarius und Violinist MARTIN SCHAUMON (ca. 1733-82) am wichtigsten. Aus den Forschungen von K. Bárdos wissen wir, daß er schon 1769 bei den Jesuiten in Tyrnau tätig war: ,fidicen Tyrnaviae simul Praefectus chori $769^{\prime .} .{ }^{51}$ Seine Funktion war also praktisch mit der des Regens chori identisch. In den Jahren 1776-82 war Schaumon „Musicus“ der Kathedrale in Pécs. ${ }^{52}$ Er hat nach Pécs einige Musikalien aus Tyrnau mitgebracht, infolgedessen wissen wir, daß Johann Fleszer auch schon 1769 Altist war, der Bassist V. A. Schorff (Scharff) und Josef Pots (Potsch) auch schon 1772 in Tyrnau gewirkt hatten. ${ }^{53}$ Vor allem ist aber Martin Schaumon Komponist der zwei in Fünfkirchen überlieferten Werke: Motetto de Sanctis („Constitues eos“) und Offertorium de Venerabile Sacramento („Lauda Sion“). ${ }^{54}$ Einige Musiker von der 1773 aufgelösten Musikkapelle der Tyrnauer Jesuiten sind wahrscheinlich weiter in Tyrnau geblieben, wir können es aufgrund des erworbenen Stadtrechtes zweier von ihnen vermuten: Joseph Mildner (sicher mit Josephus Millner identisch) aus Kreitzbier 1782 und Jozef Kolovrátek aus Velké Meziř́ičí 1783.55

Mit Martin Schaumon ist auch eine wichtige Etappe in der Musikgeschichte der Tyrnauer Jesuiten zu Ende gegangen.

51 Kornél Bárdos: Pécs zenéje a 18. században [Musik in Pécs im 18. Jahrhundert]. Budapest 1976, S. 43.

52 Ebenda, u. S. 45, 48-49, 110 .

53 Ebenda, TK Nr. 187 (NB Der Autor dieser Komposition ist nicht Lolli, sondern F. J. L. Meyer von Schauensee.) und 310 .

54 Ebenda, TK 265 und 266.

55 Vgl. Šimončič, a.a.O., S. 238 (er erwähnt aber nicht die Identität der genannten Musiker). 


\title{
Liste der Musiker
}

\section{am Tyrnauer Jesuitenkollegium bzw. an der Universität}

\begin{abstract}
Anmerkung: Nach dem Familien- und Taufname folgen die Jahre, in denen der konkrete Student in Tyrnau nachweisbar ist, dann - leider nur gelegentlich - die Angaben über Herkunft, Wohnort u. ä., schließlich gibt es Angaben (Jahreszahle, Funktionen) zu seiner Musikertätigkeit in Tyrnau. Mit einem * bezeichnete Studenten haben bei den Jesuiten studiert, doch sie haben anderswo gewirkt, d. h. sie waren nicht „Musici Collegii Academici“ oder „Templi Academici“. Die in halbfetter Schrift ausgezeichnete Musiker waren auch Komponisten.
\end{abstract}

AIGNER, Alexander 1717-19

Ungarus Nisniensis bzw. Risnensis [Tubicen]; 1717 Coll. Academici Altista; (1725 Musicus Ven. Cap. Strigon.)

BALÁSSY, Franciscus Xaverius 1757-60

BANOVICS, Josephus 1739-41 [1739] Altista

BENACZI, (Joannes) Franciscus 1724-30

1725 Templi Academ. Bassista; 1730 Bassista Templi Academ. SJ

BEN(Y)ESCHOVSKI, Martinus 1749 Altista

BERTALAMFFI, D. Georgius Ignatius Nobilis Ungarus Ovariensis 1719 Physicus

BRASIAK, D. Joannes ex Valle Dominorum 1715 Logicus

*BRECSKOCZI, Antonius (Josephus) 1714-17, Hung. ex S. Nicolao 1715 Cathedr. Eccl. Ven. Archi-Cap. Discantista; 1717 Cathedral. Eccles. Venerabil. Archi-Capituli Discantista

BRUMOVSKI, Joannes [Moravus]1701-08

1708 A.A.L.L. \& Philosophiae Baccal. Physicus

Christian, Georgius 1734-[39] Moravus Neo-tischien.

[1739] Fidicen

DEIRER (Deyrer), Joannes Josephus 1708-12 Nob. Ung. Csastensis

1712 Acad. Templi Musicus

DELPINI, D. Ignatius Wolfgangus

1717 Academici SJ Collegij Bassista, Logicus

DOPJERA, D. Joannes

1749 Bassista

(?) DRINKA, D. Franciscus

1749 Temp. Ac. Tenor.

DVORAK, Antonius

1773 Altista

ENGSZTLER, D. Joannes

1749 Logicus, Tenorista

FABER (Fabri), Christophorus

1749 Discantista 
FABRY, Joannes

1773 Altista

FANCSOVICS, D. Joannes

1749 M.B.

FLESZER (Fleser), Joannes 1769-73

[1769 Altista]; 1773 Fidicen

Fux, Michael 1730-33

1730 Discantista Templi Acad. SJ

*GARDONY, Joannes 1725-30; Nob. Ung. Bohunicensis

1725 RR..PP. Paulinorum Discantista; 1729 Templi Acad. Musicus

GAZÓ, Joannes Baptista

1749 Discantista

GerenCSERI, D. Gabriel Ignatius Nob. Ung. Banensis ex Com Trench 1737 Bassista, Logicus

GIESSER, Josephus 1737-43 Ung. Kis-Mart. ex Com. Sopr. 1737 Discantista; [1739] Altista

GRESKA, Marcellus

1773 Organista

GYURIS, Georgius

1749 Bassista

HASZIG, Michael

1730 Tenorista Templi Academ. SJ

HECKER (Hekker), Bernardus 1715-17; Moravus Wachstadiensis

1715 Discantista; 1717 Collegij Academici Tenorista

HECZER, Michael 1730

HerCZEG, Antonius Nob. Hung.

1714 Tenorista

HES(S)KY, Joannes Nobilis Moravus Nagdoczanensis

1717 Altista Academici Coll.

HEGEDÜS, Joannes

1725 Temp. Acad. Altista

HLINICZKI, Franciscus 1765

HOFFMAN, Josephus

1773 Fidicen

HORÁK (Horack), Joannes 1757-60

HORNOLUC(Z)KI, Josephus (Joannes) 1757-61

HuDADA (Chudada), Joannes Slavus

1719 [Discantista]

HuSZÁR, Nicolaus 1749-52

1749 Altista

INDRIKOVICS, Georgius 1758-60

*JANCSI, Georgius

1725 RR. PP. Paulinorum Altista

KALIVODA, Mathias

1773 Tubicen 
KARKOSS, Franciscus 1712-13

Hungarus Tyrnaviensis

KESZLER, D. Carolus

1749 Jurista, Bassista

KöNIG, D. Joannes Moravus Neo-Tischinien.,

1737 Phil. absol. Tenorista; [1739] Theol. 2di anni Tenorista

KOLOVRATEK, Joannes [Moravus]

1773 Fidicen

KrajCSOVICS, Georgius 1711-16; Panon. Csejtensis

1714 Acad. Templi Musicus

KRIDL, Ignatius

1773 Tenorista

KUNKL, Michael

1773 Huboista

KUNSST, Antonius 1737-39 Moravus Jedoviensis

[1739] Tubicen, Rhetor

LANGER, Josephus

1773 Discantista

LATKOCZI, Joannes 1712-13 Panon. Ujheliensis

1712 Discantista

LAURENSZKI, Josephus 1765

LAWICZKA (Hlawiczka), Stephanus 1729-30

1730 Discantista Templi Cathedr.

LUCSAI, D. Augustinus

1708 (Logicus)

LUKACSOVICS, Josephus

1730 Discantista Templi Academ. SJ

MAL(Y)O, Andreas 1730-32

1730 Altista Templi Academ. SJ

MATEJOVICS, Joannes 1754-60

MeCHEL, D. Alexander 1701-06

1708 (Logicus)

MERTINGER, Josephus

1749 Discantista

MEZER, Joannes 1749-53

1749 Altista

MILLER, Raphael 1746-47

1757 [Fidicen]

MiLLNER (Mildner), Josephus [Austriacus]

1773 Tubicen

MosZTENSZKY, Joannes Barth. Andreas 1725-31Ung. Halusiensis 1725 Templi Acad. Discantista; 1729 Temp. Acad. Musicus

NAROSNI, Paulus Moravus

[1739] Fidicen 
NEIBERT, Augustinus Moravus Neotics.1729-37

1730 Discantista Templi Academ. SJ

OREMA, Joannes 1765

PAL(T)ES (Bales), Josephus Panon. Ludanensis 1717-18

1717 Academ. Collegij Discantista

PAPAI, Martinus 1723-25

1725 Templi Acad. Tenorista

PAULIK, Josephus

1749 Altista

PELLER, Matthias 1711-15

Panon. Csasztensis, Coll. Academ. SJ Tyrnaviensi Altista; sowie 1714 Acad. Templi Musicus

PETRAN(I), Matthias 1707-08

PóTs (Potsch), Josephus

[1772]-73 Fidicen (Accesista)

REMETHEI, Joannes

1747-53 [Tubicen, Fidicen]; 1749 Altista

RENNER, Andreas 1749-50

1749 Tenorista; 1757 Coll. Academici SJ Musicus [Tenorista, Fidicen]

REPKOVICS, D. Josephus, Panonus Vetessensis, Coll. Academici SJ. Bassista;

1712 Physices Auditor; 1714 Theol. Mor; 1715 Sem. Mar. Szelepcs. Alumnus

REPKOVICS, Antonius 1709-14

RICHMAN (Riechman), Franciscus Thaddaeus 1711-19; Moravus Bzenecensis

1712 Coll. Academ. SJ Discantista; 1714 Templi Acad. Discantista; 1715 Altista Acad.; 1719 Physicus

RICSÁNI, Josephus Hungarus

[1739] Tubicen, Logicus

RUSICSKA, Venceslaus

1749 Bassista

SCHAUMON, Martinus

[1769]-73 Primarius; 1769 Fidicen simul Praefectus chori

$\mathrm{S}(\mathrm{CH}) \mathrm{IESL}$, Josephus

1718-19; Austriacus Leonthasiensis

SCHINDLER, Antonius Silesita

[1739] Organista, Physicus

SMIDELINI, Joannes

1749 Bassista

SCHMIDT, Joannes Hungarus

[1739] Discantista

SCHNEIDER, Thomas

1737-39 Ung. Ujbanen. ex Com. Barsch. Discantista; [1739] Discantista

SCHORFF (Scharff), Venceslaus Adalbert

[1772]-73 Bassista

SCHUSTER, Leopoldus

1712 Hungarus Comaromiensis, Coll. Acad. SJ. Tyr. Tenorista 
SKOLKA, Martinus 1709-12 Panon. Szomolanensis 1712 Acad. Templi Musicus

STIEBER, D. Antonius 1749 Physicus, Bassista

STÖCKEL, Antonius Josephus Austriacus Eberstorffensis 1715 Tenor. Acad., Logicus

SZOROCSANSKI, Franciscus

1737 Moravus Gajensis, Altista

SzTRAKOVICS, Joannes

1708 [Discantista]

TERSTYANS[Z]KI, ?

1700-01 Tubicen

UMSTATT, Josephus (Joannes) Austriacus Viennensis 1727-30 [Organista?]

VACHOVSZKI, Josephus (Joannes)

1749-57; 1757 [Fidicen]

VACHOVSSKI, Josephus 1767

VAGRI, Emericus

1725 Temp. Acad. Discantista

VALENTOVICS, Emericus 1749-53

1749 Altista

VERES, Antonius

1749 Discantista

VANTKE, Carolus Silesita

1738-[39] Fidicen, Poeta

VRANKAI, Joannes

1749 Bassista

WALTER, Josephus

1719-20 Nob. Ung. ex Valle Dominorum

ZELINKA, Franciscus

1773 Discantista

ZELINKA, Joannes Moravus

[1739] Bassista, Logicus

ZETKOVICS, Stephanus Josephus 1757-60

ZLATNIK, Josephus (Georgius) 1749-51

ZRUNEK, Josephus

1754 [Organista?]

ZUBALIK, Joannes

1749 Discantista 